\title{
Globaler flexibilisierter Kapitalismus und prozessuale Intersektionalität: Die Veränderungen nach Geschlecht und Migration in den Berufsrängen in Deutschland
}

\author{
Ilse Lenz
}

(C) Der/die Autor(en) 2020

Zusammenfassung Der Kapitalismus macht gegenwärtig weltweit eine Transformation durch und zugleich verschieben und differenzieren sich die intersektionalen Ungleichheiten nach Geschlecht, Klasse und Migration. Der Beitrag fasst zunächst wesentliche Veränderungen des Kapitalismus aus intersektionaler Sicht zusammen. Dann diskutiert er verschiedene Ansätze von Intersektionalität und bringt das Konzept prozessualer Intersektionalität ein, um die Veränderungen zu erfassen.

Darauf werden in der ersten empirischen Langzeituntersuchung für Deutschland die Veränderungen der beruflichen Ungleichheit nach Geschlecht und Migration von 1996-2011 untersucht. Während um 1996 EinwanderInnen und einheimische Frauen aus Führungspositionen weitgehend ausgeschlossen waren, waren 2011 männliche Einwanderer mit deutschem Pass dort inkorporiert. Akademische Berufe und das berufliche Mittelfeld haben sich für EinwanderInnen vor allem mit deutschem Pass geöffnet. Jedoch wird ein Drittel der Frauen mit ausländischem Pass aus Osteuropa und der Türkei in Einfacharbeit segmentiert. Geschlecht und Migration haben sich intern differenziert und wirken nun komplex in der Zuweisung von Ungleichheiten zusammen. Diese Veränderungen sollten in der Intersektionalitätsdebatte reflektiert werden.

Schlüsselwörter Arbeitsmarktungleichheit · Berufliche Segregation · Geschlecht · Intersektionalität $\cdot$ Kapitalismus $\cdot$ Migration

I. Lenz $(\bowtie)$

Fakultät für Sozialwissenschaft, Ruhr-Universität Bochum, 48101 Bochum, Deutschland

E-Mail: ilse.lenz@ruhr-uni-bochum.de 


\title{
Global flexibilised capitalism and processual intersectionality. Changes in occupational inequality by gender and migration
}

\begin{abstract}
Capitalism is manifesting a deepgoing transformation. Concurrently, intersectional inequalities are reorganised and differentiating in more complex ways. In the first part, these changes in capitalism are discussed in a global intersectional perspective. Then current approaches to intersectionality are compared and the concept of processual intersectionality is introduced to analyse the ongoing transformation.

In the second part, the first longitudinal research on changing intersectional inequality in Germany 1996-2011 is presented. Whereas in 1996, migrants and autochthonous women were excluded from management, in 2011 migrant men with German passports have been incorporated, but women are still excluded. Academic professions and midrange occupations have been opened for migrant men and women, mainly with German passport. However, many migrant women from Eastern Europe and Turkey with foreign passports are segregated into unskilled work. Gender and migration thus have been differentiated and intersectional inequalities in work are more complex. These changes should be reflected in the intersectionality debate.
\end{abstract}

Keywords Capitalism · Gender · Intersectionality · Migration · Labor market inequality $\cdot$ Occupational segregation

\section{Einleitung: Kapitalismus und Intersektionalität im Wandel}

Gegenwärtig vollziehen sich miteinander verkoppelte Umbrüche und Transformationen: Sowohl der Kapitalismus wie auch die Geschlechterordnung in den westlichen Wohlfahrtsstaaten verändern sich grundlegend (Lenz 2017). Deswegen können auch die bisherigen Theorieansätze und empirischen Strukturannahmen zum Verhältnis von Kapitalismus und Geschlecht nicht einfach fortgeschrieben werden. Für den fordistischen Kapitalismus wurde festgehalten, dass Kapitalismus, nationale Zugehörigkeit und Neopatriarchat zusammenwirken und einander stabilisieren: Während die männlichen Stammarbeiter in der Massenproduktion beschäftigt wurden, wurden Frauen vor allem als deren Hausfrauen definiert, die die unbezahlte Familienarbeit leisten sollten. Ebenso wurden EinwanderInnen vorwiegend als EinfacharbeiterInnen in der Massenproduktion eingesetzt.

Aber sind diese Aussagen für den postfordistischen Kapitalismus noch theoretisch und empirisch zutreffend und sinnvoll? Meine Grundthesen in dem folgenden Beitrag lauten, dass der Kapitalismus weltweit eine Transformation durchmacht und dass sich die intersektionalen Ungleichheiten verschieben und differenzieren. ${ }^{1}$ Da-

\footnotetext{
1 In diesem Aufsatz führe ich Debatten zur Transformation des Kapitalismus, zu prozessualer Intersektionalität und zu Veränderungen in den Berufshierarchien in Bezug auf Geschlecht und Migration zusammen. In Teilen geht er auf Lenz (2017, 2018, 2019a, 2019b, 2020) zurück und führt diese Überlegungen weiter. Dort wurde auch die Fachliteratur zu diesen Fragen aufgeführt und zusammengefasst (vgl. ibid.), was in diesem Rahmen nicht möglich war.
} 
bei besteht keine einseitige Determination durch den Kapitalismus, sodass seine Veränderungen notwendig zu bestimmten Ungleichheitsstrukturen führen würden, sondern es zeigen sich komplexe und weltweit divergierende Wechselverhältnisse zwischen beiden. Deswegen erfordern die gegenwärtigen Transformationen sowohl eine kritische Reflektion zentraler Theorieansätze wie auch empirische Untersuchungen, die diese Veränderungen und die Wechselverhältnisse zwischen Kapitalismus und intersektionalen Ungleichheiten fokussieren.

Eine Reihe unterschiedlicher Ansätze diskutieren die gegenwärtigen Transformationen des Kapitalismus, teils auch in Bezug auf das Geschlechterverhältnis. Aber eine theoretische Synthese steht einstweilen aus und es ist fraglich, ob sie auf dem gegenwärtigen Forschungsstand schon möglich ist. ${ }^{2}$ Im ersten Teil des Beitrags versuche ich deswegen einstweilen, den theoretischen und empirischen Rahmen der gegenwärtigen Debatte zur Transformation des Kapitalismus und der Geschlechterordnungen zu umreißen. Zunächst möchte ich einige Eckpunkte der Debatten zu Kapitalismus und Geschlecht zusammenfassen und lege dem eine intersektionale Sicht zugrunde. Dabei wird die innovative und dynamische Kraft des Kapitalismus herausgearbeitet, der geschlechtliche und ethnische Ungleichheiten sowohl aufbricht und reorganisiert, wie er sie auch neu fixiert und verankert. Da diese wechselwirkenden Ungleichheiten theoretisch unter Intersektionalität gefasst werden, will ich darauf das Spektrum der verschiedenen Intersektionalitätsansätze kritisch zusammenfassen. Im ersten Teil dieses Beitrags werden also Ansätze zum postfordistischen Kapitalismus und zu Intersektionalität zusammengeführt.

Im zweiten Teil werde ich in einer Fallstudie untersuchen, ob in Deutschland empirische Veränderungen in beruflichen Zugängen nach Geschlecht und Migration festzustellen sind, also die intersektionalen Ungleichheitsstrukturen gleichblieben oder sich verschoben haben. Betrachtet wird der Zeitraum von 1996-2011, in dem die Tendenzen zum globalisierten und flexibilisierten Kapitalismus deutlich zunahmen. Wie wurden verschiedene Gruppen von Einheimischen und EinwanderInnen auf die unterschiedlichen Berufshierarchien - zwischen Management und Einfacharbeit - positioniert und hat sich das verändert? Die Fallstudie beruht auf Auswertungen in Kreuztabellen auf Grundlage des Mikrozensus, also der größten verfügbaren Datenbasis zu beruflicher intersektionaler Ungleichheit. Sie stellt zum einen ein Beispiel für die Veränderungen von Kapitalismus und Intersektionalität in den letzten Jahrzehnten dar. Zum anderen aber bietet sie die erste empirische Langzeitstudie für Deutschland, in der die erheblichen intersektionalen Veränderungen in den beruflichen Chancen und Ausschlüssen erfasst werden und bisherige Alltagsannahmen über eine durchgehende und gleichbleibende Ausgrenzung nach Migration widerlegt werden.

Das unerwartete Ergebnis war, dass der vorige weitgehende Ausschluss von Frauen und EinwanderInnen aus den Spitzenberufen und der lohnarbeitenden Mitte

\footnotetext{
2 Vgl. zur Kapitalismusdiskussion u. a. Aulenbacher et al. (2015); Becker et al. (2020); Dörre et al. (2012); Kocka (2014); Kößler (2013); Scheele und Wöhl (2018); Wallerstein et al. (2013). Zum Postfordismus vgl. u. a. Atzmüller et al. (2015); Aulenbacher et al. (2015); Edgell et al. (2016); Gottfried (2013); HirschKreiensen und Minssen (2013), Lenz et al. (2017), Lenz (2017); Pries (2010); Reckwitz (2017); Wallerstein et al. (2013). In diesem Rahmen kann ich nicht auf den Finanzkapitalismus eingehen.
} 
durchbrochen wurde: Zum Beispiel ist der Anteil von Führungskräften unter deutschtürkischen Männern 2011 ebenso hoch wie unter langheimisch Deutschen. Auch der Anteil von Fachkräften unter den EinwanderInnen ist gestiegen. Allerdings befinden sich große Gruppen von EinwanderInnen weiterhin in den Arbeiterberufen und vor allem Frauen mit ausländischem Pass werden in die Einfacharbeit segmentiert. Wo Menschen in den beruflichen Hierarchien platziert werden, lässt sich also nicht mehr unmittelbar von Geschlecht oder Migration ableiten. Das zählebige Gastarbeiterbild von den EinwanderInnen geht an den komplexer gewordenen Ungleichheitsstrukturen vorbei - ebenso wie das Bild der Hausfrau für die sich differenzierende Lage der einheimischen und eingewanderten Frauen. Vielmehr zeigen sich komplexe Wechselwirkungen zwischen Geschlecht und Migration: Denn innerhalb beider Strukturkategorien sind Differenzierungen zu beobachten, die nun in komplexen Ungleichheiten resultieren: So sind einige einheimische und eingewanderte Frauen an der beruflichen Spitze und in der lohnarbeitenden Mitte zu finden, und dennoch sind die Berufe weiterhin geschlechtlich segregiert und die Migration wirkt sich auf die Positionierung in den Berufsrängen aus. Wie ich unten im Einzelnen ausführe, wurden die Ungleichheiten reorganisiert und differenziert.

Hinsichtlich Intersektionalität beziehe ich die Fallstudie auf den theoretischen Rahmen zurück. Denn ihre Ergebnisse widersprechen einem Teil der Intersektionalitätsansätze, die von einer durchgehenden Privilegierung einheimischer oder „,weißer“ Personen ausgehen. Deswegen soll die Intersektionalitätsdebatte daraufhin betrachtet werden, inwieweit sie offen für sozialen Wandel ist. In diesem Zusammenhang schlage ich den Ansatz prozessualer Intersektionalität vor.

\section{Die Transformation des Kapitalismus und soziale Ungleichheiten}

Der Kapitalismus steht im Wechselverhältnis zu sozialen Ungleichheiten wie Geschlecht und Ethnizität: Er legt sie systematisch zugrunde, baut sie in seine Wirkungsweisen ein und trägt so bei, sie zu reproduzieren. Allerdings behaupten diese weiterhin eine relative Eigenständigkeit und sind nicht einfach vom Kapitalismus abzuleiten. Wie ist dieses Wechselverhältnis zu verstehen? Dafür ist es sinnvoll, die Debatten um Kapitalismus und Geschlecht und die postfordistische Transformation des Kapitalismus zusammenzuführen, indem einige ihrer wesentlichen Ergebnisse festgehalten werden.

Der Kapitalismus bezieht sich auf vorgängige Ungleichheiten, indem er sie reorganisiert und so in seine Wirkungsweisen einbaut. Beispielhaft zeigt sich das an der Verschränkung von Kapitalismus und Patriarchat. In den Forschungen zu Arbeit und Geschlecht wurde ein erweitertes Verständnis des Kapitalismus herausgearbeitet, das die Produktion und die Reproduktion im Kapitalismus in ihren wechselseitigen Verschränkungen sichtbar macht und zugrundelegt (vgl. Becker et al. 2020; BeckerSchmidt und Knapp 2000; Lenz et al. 2017; Lenz 2017; Scheele und Wöhl 2018). Dabei wurde die unbezahlte Versorgungsarbeit zur Reproduktion der ArbeiterInnen mit einbezogen: Sie ist dem Kapitalismus untergeordnet, wurde aber im modernen Neopatriarchat per Geschlecht den Frauen zugewiesen. Diese wurden als Hausfrauen definiert, die abhängig vom Lohnarbeiter/Ehemann die Familienarbeit leisten 
sollen. Demnach wirken zwei ungleichheitsgenerierende Strukturen, also Kapitalismus und Neopatriarchat, zusammen. Dem Kapitalismus subsumiert und durch ihn re-organisiert ist ein breites Spektrum entlohnter und bezahlter, freier und unfreier Arbeitsverhältnisse (Lenz 2017).

Während der Kapitalismus in einem Wechselverhältnis zu sozialen Ungleichheiten steht, so ist dies nicht als einlinige Bestärkung zu verstehen. Vielmehr zeigen sich Konvergenzen und Widersprüche zwischen dem Kapitalismus und anderen Ungleichheitssystemen wie dem Neopatriarchat. Aus der Sicht der Kritischen Theorie hat Regina Becker-Schmidt in ihrem klassischen Ansatz zur doppelten Vergesellschaftung der Frau auf diesen Widerspruch hingewiesen: Während Frauen durch das Patriarchat aufgrund ihres Geschlechts in die unbezahlte Hausarbeit vergesellschaftet werden, werden sie auf dem kapitalistischen Arbeitsmarkt als potenziell freie Arbeitskraft aufgenommen. So zeigt sich ein Widerspruch zwischen der geschlechtlichen personalen Unterordnung in der Familie und der Freisetzung für den Arbeitsmarkt (Becker-Schmidt und Knapp 2000).

Die Nation vermittelt ebenfalls Ungleichheiten zwischen langheimischen Mitgliedern und EinwanderInnen, was in dem Citizenship-Ansatz aufgezeigt wurde. Zum Beispiel wurde in Deutschland der Zugang zu Arbeitsplätzen primär deutschen StaatsbürgerInnen zuerteilt, sodass Frauen mit deutschem Pass gegenüber männlichen Einwanderern mit ausländischem Pass rechtlich bevorzugt wurden. Während die Nationalität ebenfalls mit dem Kapitalismus und dem Patriarchat zusammenwirkt, steht sie zu diesen Kräften auch im Widerspruch. Denn es handelt sich um Ungleichheitsverhältnisse, die in Wechselwirkung stehen, aber relative Autonomie behaupten (vgl. Lenz 1995). Der Ansatz der Inklusion/Exklusion knüpft an diesen Widersprüchen an und entwickelt daraus ein relationales Konzept der Migrationsforschung (Ataç und Rosenberger 2013).

Nach der Kritischen Theorie verarbeiten die Menschen diese Widersprüche in subjektiven Ambivalenzen. Sie denken und handeln danach nicht einfach entsprechend ihrer sozialen Positionierung etwa als Managerin oder Migrant am Fließband, sondern sie setzen sich kritisch damit auseinander und schaffen teils neue ambivalente Subjektentwürfe, in denen sie die strukturellen Ungleichheiten teils verinnerlichen, teils sich widerständig, grenzüberschreitend oder kritisch verhalten (BeckerSchmidt und Knapp 2000). Laut einer qualitativen Untersuchung wehrten sich zum Beispiel linke kurdische Flüchtlinge dagegen, dass ihnen eine patriarchale muslimische Identität zugeschrieben wurde. Vielmehr hatten sie in ihrer Sicht bereits vor der Flucht gegen patriarchale Normen gekämpft und wünschten sich in ihrem Subjektentwurf gleichheitliche Beziehungen zu Frauen im Alltag und in der Politik (vgl. Ghaderi 2014).

So eröffnet die Kritische Theorie ein komplexes Verständnis zu Kapitalismus und Ungleichheiten, das erlaubt, sowohl deren wechselseitige Konstitution und Koevolution wie auch deren Widersprüche wahrzunehmen und zu begreifen (Becker-Schmidt und Knapp 2000). Jedoch betrachtet sie die Ungleichheiten im Kapitalismus eher als langzeitlich wirkend und stabil.

Während der Kapitalismus Ungleichheiten zugrundelegt und sie in seine Wirkungsweise einbaut, bricht er sie auch kontinuierlich wieder auf. In diesem Sinne wirkt er als innovatorische und reorganisierende Kraft. Stellt er doch eine Kraft 
sowohl der gesellschaftlichen Veränderungen wie auch der dauernden Re-Organisation von Ungleichheiten dar. Gegenwärtig wird eine tiefgreifende Transformation des Kapitalismus festgehalten, die weithin als Postfordismus bezeichnet wurde. Die Debatte dazu ist lang und weit gefächert. ${ }^{3}$ Betont werden die Trends zur Vermarktlichung und Ökonomisierung der Produktion, zu ihrer räumlichen und zeitlichen Reorganisierung und ihrer Flexibilisierung sowie zur Digitalisierung und Kulturalisierung. Diese stützen sich auf digitale Kommunikations- und Informationstechnologie, die die Koordination und Steuerung hochkomplexer Prozesse raumübergreifend ermöglichen.

Diese Trends sind miteinander gekoppelt: Die Ökonomisierung und Vermarktlichung der Produktion und damit auch der Arbeitskraft umspannt den Erdball. Im Globalen Norden ${ }^{4}$ zeigte sich ein rasches Anwachsen der Frauenerwerbstätigkeit oder anders gesagt der Kommodifizierung der Arbeitskraft von Frauen. Während sie im Fordismus eher auf ungelernten Arbeitsplätzen und vor der Familiengründung beschäftigt wurden, zogen Frauen nun auch in qualifizierte berufliche Positionen ein und eröffneten sich lebenslange Berufslaufbahnen. Dieser Artikel wird darlegen, dass sich ähnliche Trends unter den EinwanderInnen zeigen. Betriebliche Diversitätsansätze richten sich auf eine effektivere Nutzung der Arbeitskraft von Migrantinnen wie auch queeren Personen und richten dazu auch Maßnahmen zu Aufstieg und Antidiskriminierung ein. Die vorigen klaren Segmentierungen und Ausgrenzungen nach Geschlecht und Migration werden nun komplexer.

Zugleich wurden im Globalen Süden im Zuge der abhängigen Industrialisierung Millionen von ArbeiterInnen, insbesondere junge Frauen vom Land oder aus dem informellen Sektor, in den neuen Weltmarktfabriken beschäftigt. Dabei ist freie Lohnarbeit nur ein Pol im Spektrum der obigen Beschäftigungsformen zwischen flexibilisierter, unfreier und Zwangsarbeit. Diese Formen verlaufen zwischen freiem Arbeitsvertrag mit existenzsichernden Löhnen, flexibilisierter oder irregulärer Arbeit, marktvermittelter Produktion für internationale Konzerne wie Heimarbeit im Textilsektor oder formeller Selbstständigkeit und unfreier Arbeit. ${ }^{5}$ Diese unterschiedlichen, miteinander verflochtenen Arbeitsformen sind insgesamt dem Kapitalismus subsumiert: Direkt durch Arbeitsverträge, indirekt durch marktvermittelte Formen wie Zuliefererproduktion oder Soloselbstständigkeit (Lenz 2010). Dies zeigt sich etwa an den globalen Produktionsketten in der Elektronik- und Bekleidungsindustrie. So wurden beispielsweise bei Spitzenblusen die Designs im Globalen Norden durch KreativarbeiterInnen in der Modeindustrie entworfen, während die Blusen in Bangladesh genäht und die Spitzen von niedrig entlohnten Heimarbeiterinnen hergestellt wurden. Auch in seiner postfordistischen Transformation bezieht sich der

\footnotetext{
3 Vgl. Fußnote 2.

4 In Anlehnung an die Globalisierungsdebatte übernehme ich hier regionale Bezeichnungen zur Charakterisierung der globalen Machtverhältnisse. Der globale Norden steht für die postindustriellen kapitalistischen Gesellschaften und der globale Süden für die peripheren Gesellschaften in Afrika, Asien und Lateinamerika.

5 Vgl. u. a. Lenz (2010). Die unfreie Arbeit wie Zwangsarbeit und Zwangsprostitution umfasst nach Schätzungen der ILO immerhin ca. 20 Mio. (ILO 2014, S. 7).
} 
Kapitalismus also auf vorgängige Ungleichheiten nach Geschlecht, Migration und „Rasse“, aber er reorganisiert sie in komplexeren, weltweit verflochtenen Formen.

In dieser reorganisierenden Übernahme sozialer Ungleichheiten nimmt der Kapitalismus Ungleichheitsideologien auf, wie dies etwa in Bezug auf eine ihnen zugeschriebene geringere Qualifikation und Arbeitsmotivation von Frauen oder auch von EinwanderInnen im Fordismus geschah. Diese Ideologien werden aufgenommen und angepasst, um die hierarchischen Positionierungen in der Arbeit zu legitimieren. Sie können sich aber ändern und modernisiert werden. Dabei wirken sich auch die gesellschaftliche Modernisierung und die Frauenbewegung aus. Wurden die Frauen im Fordismus als Mütter und Hausfrauen der männlichen Ernährer definiert, so stehen sie nun teils für Diversity im Betrieb, oft unter dem Leitwort einer vermeintlichen ,weiblichen Begabung“. Bei EinwanderInnen ist eine ähnliche Umdeutung vom Bild des ,ungelernten Gastarbeiters“ zum Träger kulturspezifischer soft skills zu beobachten.

Parallel zur Ökonomisierung und Flexibilisierung der Lohnarbeit wie auch der Modernisierung der Geschlechterbilder hat sich im Globalen Norden jedoch kein neues konsistentes Leitmuster der Reproduktion der Arbeitskräfte etabliert (vgl. Aulenbacher et al. 2014; Lenz 2017). Vielmehr wurde die Reproduktion dort erneut tendenziell zur „Privatsache“ erklärt und familiale Vielfalt oder auch der Singlestatus legitim. In den 1990er-Jahren sind die Geburtenraten im Globalen Norden weithin unter das Niveau der Bestandserhaltung gefallen, auch wenn sie in einzelnen Ländern wie in Deutschland wieder leicht wachsen (vgl. u. a. http://www.oecd.org/els/family/ database.htm, Zugegriffen 02.09.2020). Die Kommodifizierung der Arbeitskraft von Frauen wird abgestützt durch fragmentierte soziale Infrastruktur im Bereich der Kinder- und Altenbetreuung und informelle Pflegearbeit von MigrantInnen. Ansonsten wird auf weitere unbezahlte Versorgungsarbeit der nun überwiegend erwerbstätigen Frauen gesetzt u. a. durch Pflegeurlaube, Teilzeitarbeit usw. In der Folge scheint der Postfordismus durch Krisen der Reproduktion gekennzeichnet.

Auch zeigen sich Veränderungen der Einwanderung und der Migrationspolitik (vgl. Schwenken 2018). Man könnte sie als Übergang von dem fordistischen Leitbild des „Gastarbeiters“ in der Migrationspolitik zu dem des ,qualifizierten $\mathrm{Hu}$ mankapitals“ zusammenfassen. Während die Arbeitsmigration in die fordistische Massenproduktion in Deutschland vor allem aus Südeuropa und der Türkei erfolgte, nimmt die qualifizierte Migration aus dem Globalen Süden und insbesondere dem Nahen Osten zu.

Im Globalen Süden haben sich aufgrund verschiedener Entwicklungen die Migrationsformen und -motive ebenfalls verändert. Die ansteigende Allgemeinbildung drückt sich angesichts fehlender Chancen im Herkunftsland auch in einer Zunahme qualifizierter internationaler Migration aus. Militärische Konflikte und failed states führten zu Fluchtmigration, wobei die große Mehrheit der Flüchtlinge weiterhin im Globalen Süden verbleibt. Frauen machen etwa die Hälfte der internationalen Migration aus, was als Feminisierung der Migration beschrieben wurde (vgl. Schwenken 2018).

In diesem Abschnitt habe ich versucht, die Transformation des Kapitalismus in einigen Grundtendenzen zu umreißen, und argumentiert, dass er soziale Ungleichheiten wie Geschlecht und Ethnizität sowohl stabilisiert wie auch aufbricht und ver- 
ändert. Diese sind relativ eigenständig und beruhen ihrerseits auf Systemen wie dem Neopatriarchat und der Ethnizität der Nation, die ethnische und rassistische Ausgrenzung abstützt. Auch sie verändern sich durch die reflexive Modernisierung, die Globalisierung und soziale Bewegungen. So ist fraglich, ob gegenwärtig von einem Neopatriarchat als stabilem System oder nicht eher von Geschlechterasymmetrie zu sprechen ist (vgl. Lenz 2019b). Diese Veränderungen könnten sich wechselseitig verstärken oder aber Strategien der Restabilisierung der Ungleichheiten hervorrufen. Inwiefern nimmt der Ansatz der Intersektionalität, der diese Ungleichheiten und ihre Wechselwirkungen untersucht, diese Spannungen und Veränderungen auf?

\section{Varianten von Intersektionalität und prozessuale Intersektionalität}

Vor dreißig Jahren begründete Kimberlé Crenshaw den Intersektionalitätsansatz, um die Wechselwirkungen zwischen Geschlecht und „Rasse“ zu analysieren (Crenshaw 1989; Hill Collins und Bilge 2016; Meyer 2017; Gunda-Werner-Institut 2019). Dieser verbreitete sich rasch international in Wissenschaft und Praxis. Von der UN bis zu linksfeministischen Netzwerken vor Ort wurde er aufgegriffen und weitergeführt.

Der Ansatz hat sich differenziert und in verschiedene Perspektiven verzweigt (vgl. u. a. Lutz et al. 2013). Nach dreißig Jahren scheint eine Reflektion und Systematisierung dieser Varianten von Intersektionalität sinnvoll (vgl. dazu Lenz 2019a). Aufbauend auf diesen Überlegungen geht es im Folgenden um die gegenwärtigen theoretischen Sichtweisen auf Intersektionalität und nicht um empirische Verhältnisse. Ich schlage vor, zwei Kriterien für eine solche Systematisierung heranzuziehen: Zum einen ist zu fragen, an welche Theorien sie sich anlehnen, wie zum Beispiel an postkonstruktivistische, identitätspolitische oder strukturtheoretische Ausrichtungen. Zum Zweiten geht es darum, welche unterschiedliche Dimensionen von Intersektionalität sie fokussieren: Zielen sie auf die diskursive und symbolische Dimension etwa bei der Repräsentation Schwarzer Frauen oder auf die sozialstrukturelle Dimension zum Beispiel bei Ungleichheit auf dem Arbeitsmarkt. Wenn man beide Kriterien einbezieht, kann man nach den zugrundeliegenden Theorierichtungen zwischen diskursiver, struktureller und positionaler Intersektionalität unterscheiden (ibid.). Die beiden ersteren Zugänge fokussieren die Herrschaftsverhältnisse und Machtmechanismen, die intersektionale Ungleichheiten schaffen, begründen und legitimieren. Im Folgenden werden diese drei Ansätze sehr knapp umrissen, wobei auf ihre Differenzierungen in diesem Rahmen leider nicht eingegangen werden kann.

Diskursive Intersektionalität untersucht Machtdynamiken, Dominanzstrategien und Ungleichheiten im Feld der Anerkennung, des symbolischen Kapitals und der Kultur. Sie lehnt sich meist an diskurstheoretische und postkonstruktivistische Ansätze vor allem nach Judith Butler und Michel Foucault an. Sie hat vor allem die Verbindungen von Rassismus, Heteronormativität und Sexismus herausgearbeitet, während die Klasse eher links liegengelassen wurde (vgl. u. a. Hark und Villa 2017; Dietze 2017). Danach ermöglicht etwa die Konstruktion der traditionellen Migrantin in den einheimischen Medien sowohl, diese als ,zurückgeblieben“ abzuwerten wie auch die eigene Gruppe oder sich selbst als emanzipiert und modern aufzuwerten. 
Strukturelle Intersektionalitätsansätze untersuchen, welche Strukturen und Herrschaftssysteme den intersektionalen Ungleichheiten zugrunde liegen und sie miterzeugen: Nach der feministischen Kritischen Theorie resultieren sie aus den grundlegenden „Achsen der Ungleichheit“ der Moderne, also Patriarchat, Kapitalismus und Imperialismus (Klinger et al. 2007; Winker und Degele 2009). Dieser Strukturansatz arbeitet, wie gesagt, die Spannungen und Widersprüche zwischen diesen Achsen heraus, wie etwa zwischen der häuslichen Unterordnung von einheimischen und eingewanderten Frauen durch das Patriarchat und ihrer Nutzung als Arbeitskraft durch den Kapitalismus. Diese Widersprüche treten etwa bei der Ausbeutung von Einwanderinnen als irreguläre Arbeiterinnen im Haushalt zutage (vgl. Lutz und Amelina 2017).

Die Veränderungen dieser Herrschaftsstrukturen lassen also auch Veränderungen der intersektionalen Verhältnisse erwarten. Dementsprechend kann sich die Transformation des Kapitalismus so auswirken, dass bisherige Ungleichheiten in der globalen Arbeitsorganisation oder in der vergeschlechtlichten Migration reorganisiert oder neu formiert werden. Die diskursiven und strukturellen Perspektiven stehen nicht gegeneinander, sondern ergänzen sich vielmehr.

Demgegenüber leiten positionale Intersektionalitätsansätze wechselwirkende Ungleichheiten direkt von gesellschaftlichen Strukturkategorien wie Geschlecht oder „Rasse“ ab, die ungleiche Gruppenpositionen begründen: Eine weiße Frau wäre danach gegenüber einem schwarzen Mann privilegiert, um ein geläufiges Beispiel heranzuziehen. Die Kategorien führten also zu gemeinsamen sozialen Positionen, die wiederum auf gemeinsame Identitäten hinwirken. Die zugrundeliegenden Strukturen und Herrschaftssysteme werden wenig thematisiert - und noch weniger deren mögliche Veränderungen wie etwa die Transformation des Kapitalismus. Da die Erklärung von Ungleichheiten direkt aus diesen Positionen abgeleitet wird, spreche ich von positionaler Intersektionalität.

Angesichts der antirassistischen Bewegung in Europa und den USA, die infolge der Black-Lives-Matter-Mobilisierung aufgeflammt ist, wird vor allem positionale Intersektionalität in der gesellschaftspolitischen Debatte wahrgenommen und teils mit dem Ansatz insgesamt gleichgesetzt. Allerdings wurde auch eine Reihe von Einwänden vorgebracht (vgl. u. a. Hark und Villa 2017; Lenz 2019a). Danach werden Ungleichheiten vor allem an Diskriminierungen nach Geschlecht und Rasse festgemacht, während Klasse weitgehend ausgeblendet wird. Teils wird davon ausgegangen, dass mit bestimmten Positionen (,männlich“, ,,weiß“) durchgehend mehr Privilegien verbunden sind. Diese erscheinen letztlich als eher diffuse Ressourcen; tendenziell hat die Rede von Privilegien die Analyse von empirischen Strukturen und Widersprüchen abgelöst. Die kategorial vermittelte Position bestimmt in dieser Sicht auch unmittelbar das Bewusstsein, etwa von weißen Frauen und Männern, wobei meist nicht nach Klasse differenziert wird. Ambivalenzen werden kaum thematisiert. Schließlich wird von einer Langzeitwirkung zumindest der Kategorie „Rasse“, teils seit dem Kolonialismus, ausgegangen. Es werden also stabile intersektionale Ungleichheiten angenommen.

Eine weitere Variante ergibt sich aus der Frage, ob die jeweiligen Zugänge zu Intersektionalität eher eine statische Sicht auf stabile Ungleichheiten haben, oder ob sie Veränderungen oder Rekonfigurationen intersektionaler Ungleichheiten wahrneh- 
Tab. 1 Varianten von Intersektionalität

\begin{tabular}{lll}
\hline & Diskursiv & Strukturell \\
\hline Statisch & Diskursive Intersektionalität & Strukturelle Intersektionalität \\
& Positionale Intersektionalität & Positionale Intersektionaliät \\
Prozessual & Diskurstransformation & Strukturelle Transformation \\
\hline
\end{tabular}

men und bearbeiten. Bisher wurden Prozessperspektiven kaum eingebracht, obwohl sich gegenwärtig intersektionale Ungleichheiten in der Globalisierung und reflexiven Modernisierung verändern.

Ich möchte deswegen den Arbeitsbegriff der prozessualen Intersektionalität als eine weitere Variante einbringen. Er fokussiert, wie intersektionale Ungleichheitsverhältnisse sich durch strukturellen, institutionellen oder kulturellen Wandel verändern: Sie können rekonfiguriert, differenziert oder auch tendenziell abgebaut werden. Es handelt sich um eine empirische Perspektive, nicht um ein normatives teleologisches Konzept, das von der Vorstellung eines zunehmenden Fortschritts geleitet wäre. Die Veränderungen können durch eine Reorganisierung der Herrschaftsverhältnisse wie auch durch soziotechnische Innovation wie etwa die Digitalisierung oder durch den Wandel von Bewusstsein und Praktiken angestoßen sein; meist wirken diese Faktoren zusammen. Ich schlage vor, die unterschiedlichen Ansätze entlang der Achsen dieser beiden Fragen wie in der folgenden Übersicht zu verorten (Tab. 1).

Wie in der folgenden Fallstudie erläutert wird, haben sich die Chancen von Menschen aus Arbeiter- und Einwandererfamilien in Deutschland u. a. durch neue Personalkonzepte des globalisierten Kapitalismus und vor allem durch ihre hohe Bildungsund Berufsmotivation verändert und sie haben sich in sozialen Bewegungen dafür eingesetzt.

\section{Vom Ausschluss zur selektiven Inkorporation: Die Veränderungen nach Geschlecht und Migration in den Berufsrängen in Deutschland}

Die Transformation des Kapitalismus wirkt auf Ungleichheiten in Produktion und Reproduktion ein und damit auf die intersektionale Ungleichheit nach Geschlecht und Migration, wie meine Leitthese lautet. Wie oben angesprochen, will ich nun an einer Fallstudie zu Deutschland untersuchen, ob parallel zu dieser Transformation empirische Veränderungen in dem Zugang zu Einschlüssen und Ausschlüssen nach Geschlecht und Migration in der Lohnarbeit festzustellen sind. ${ }^{6}$

Dazu bringe ich die Ergebnisse einer laufenden Untersuchung zu den sich verändernden Zugängen zu den hierarchischen Berufsgruppen nach Geschlecht und Migration ein. Sie beruht auf einer Auswertung des Mikrozensus von 1996-20117

\footnotetext{
6 Während sich ein paralleler Verlauf konstatieren lässt, lässt sich daraus keine kausale Beziehung ableiten. Wohl aber reicht er dazu hin, weitere Hypothesen für folgende Untersuchungen zu fundieren, die den möglichen Wechselwirkungen nachgehen.

7 Ausgewertet wurden die Scientific-Use-Files des Mikrozensus (anonymisierte 70\%-Unterstichprobe) der Jahrgänge 1991, 1996, 2000, 2005, 2008, 2009, 2010, 2011. Quelle: FDZ der Statistischen Ämter des Bundes und der Länder, Mikrozensus 1991-2011, dem herzlich gedankt sei; eigene Berechnungen.
} 
und ist in mehrerlei Hinsicht neu: Sie betrachtet die Ungleichheiten nach Geschlecht und Migration in ihrer Wechselwirkung über etwa zwanzig Jahre. Es handelt sich in diesem Sinne um die erste langzeitliche intersektionale Studie für Arbeitsmarkt und Beruf in Deutschland. ${ }^{8}$

In Bezug auf die Variable Migration wurde der immer noch verbreitete Dualismus zwischen Einheimischen und MigrantInnen vermieden. Vielmehr wurde zum einen differenziert nach langheimisch Deutschen, Deutschen mit Migrationshintergrund und EinwanderInnen mit ausländischem Pass, also nach Staatsbürgerschaft/ Migrationsstatus. Die regionale Herkunft diente als zweites Kriterium der Gruppenbildung.

Ich will nun kurz auf das Auswertungsverfahren eingehen. Vor allem aus folgenden Gründen wurde der Mikrozensus ausgewählt (vgl. Bandorski et al. 2009): Erstens bietet er eine umfassende Datenbasis von EinwanderInnen aus verschiedenen Herkunftsländern. So ermöglicht er, jeweils ein hinreichend großes Sample einzelner Herkunftsgruppen zusammenzustellen, um Aussagen über deren Verteilung über die Berufshierarchien zu treffen. ${ }^{9}$ Dementsprechend wurden große Herkunftsgruppen ausgewählt und ausgewertet, die mit mindestens 400 Personen im Mikrozensus vertreten sind; meist liegen die Angaben aber erheblich höher: Es handelt sich um EinwanderInnen aus Italien, Ex-Jugoslawien, der Türkei, Polen und den GUSStaaten. Weiterhin wurden sie in zwei regionale Gruppen der EinwanderInnen aus Südeuropa (Italien, Ex-Jugoslawien, Türkei) und Osteuropa (Polen, GUS-Staaten) zusammengefasst.

Ein weiterer Grund war, dass der Mikrozensus ab 2005 die Unterscheidung nach dem Migrationsstatus aufgenommen hat: Damals führte er den Begriff des Migrationshintergrundes ein und unterscheidet seitdem nach Personen mit ausländischer Staatsangehörigkeit und/oder Eingebürgerte oder Deutsche mit einem ausländischen Elternteil. Die beiden letzteren Gruppen bezeichne ich hier aus pragmatischen Gründen als EinwanderInnen mit deutschem Pass. Von Teilen der EinwanderInnen wird der Begriff Migrationshintergrund zunehmend als ausgrenzend aufgefasst. Jedoch zeigt er zumindest auf, dass er nun etwa ein Viertel der Bevölkerung einschließt und Deutschland ein Einwanderungsland ist. Ich verwende ihn nur zur Beschreibung eines institutionellen Status, da passendere Begriffe fehlen.

Der Migrationsstatus vermittelt jeweils unterschiedliche Sicherheit im Aufenthalt und der Lebensplanung. Deutsche Staatsbürgerschaft eröffnet mehr Chancen auf Teilhabe durch einen gesicherten Aufenthalt und eine langfristige Lebensplanung (vgl. Ataç und Rosenberger 2013; Söhn 2011). Im Ergebnis erwies sich der Migrationsstatus als ähnlich wichtig für Einschlüsse und Ausschlüsse von Berufschancen wie das Herkunftsland. Diese doppelte Perspektive auf Herkunftsregion und Migrationsstatus trägt potenziell zu einer Differenzierung und der Untersuchung und De-

Die Daten für 1991 waren noch stark von der deutschen Vereinigung 1990 beeinflusst und wurden hier deswegen ausgelassen. Die Auswertung wurde in der ersten Stufe von Shih-cheng Lien, Sonja Teupen und darauf von Werner Voss durchgeführt, denen ich hier herzlich danken möchte.

8 In diesem Beitrag wird eher die heutige Lage vorgestellt; zum Verlauf seit 1996 und der parallelen Entwicklung in der Bildung vgl. Lenz (2020).

9 Das bildet einen Vorzug gegenüber dem Sozioökonomischen Panel (SOEP), das seinerseits detaillierte, auch subjektorientierte Auswertungen ermöglicht. 
konstruktion ethnisierender und kulturalisierender Sichtweisen bei: Denn wenn sich die Verhältnisse z. B. zwischen Deutsch-PolInnen und PolInnen klar unterscheiden, hat die „Herkunftskultur“ offensichtlich nicht die aussschlaggebende Bedeutung, die ihr oft zugeschrieben wird. Wesentlich für die soziale und subjektive Verortung ist nicht eine - oft konstruierte und ethnisierte - „Kultur“, sondern was sich in den individuellen Biografien zwischen der Herkunftsregion und der „Lebensregion“ Deutschland abgespielt hat und was heute geschieht. Der Mikrozensus ermöglicht also, die Teilhabe im Beruf nach Herkunftsland wie auch nach Staatsbürgerschaft/ Migrationsstatus für alle in Deutschland lebenden Menschen zu vergleichen. In der Fallstudie wird eine Sonderkonstruktion von MigrantInnen als spezieller Einheit vermieden, indem alle Gruppen, auch die langheimisch Deutschen, eingeschlossen wurden. So wird ein Gesamtbild der Teilhabe nach Geschlecht und Migration statt einer Sonderbetrachtung von MigrantInnen ermöglicht.

Die Leitfrage der Fallstudie lautet, wie sich die Gruppen auf die unterschiedlichen Berufshierarchien - zwischen Management und Einfacharbeit - verteilen. Diese Hierarchien beinhalten Ungleichheiten in Bezug auf die Entlohnung und Beschäftigungssicherheit wie auch die Arbeitsinhalte und Qualifikationen. Dabei stellt sich das zusätzliche Problem, dass EinwanderInnen teils nicht qualifikationsangemessen beschäftigt werden. Unter anderem wird eine Ausbildung außerhalb Deutschlands teils nicht entsprechend anerkannt und auch informelle Skills werden mitgenutzt, aber nicht entsprechend eingestuft. Leider kann dem in diesem Rahmen nicht nachgegangen werden. Denn es geht zunächst um eine Beschreibung der intersektionalen Ungleichheit in den Berufshierarchien auf gesamtgesellschaftlicher Ebene und (noch) nicht um deren Erklärung.

Weiterhin ergaben sich große Unterschiede auch innerhalb der jeweiligen Gruppe: So treten innerhalb der Deutsch-Türken relativ hohe Anteile von Führungskräften wie auch von Arbeitern auf. Im Kern geht es um den Vergleich von Prozessen von Inklusion und Exklusion, die Personen in Strukturen betreffen, und nicht von unterschiedlichen Gruppen. Solche Strukturen sind etwa die kapitalistischen Betriebe mit ihrer Plazierung (Allokation) von bestimmten Personen auf bestimmte Jobs, die ungleiche geschlechtliche Arbeitsteilung zwischen Heim und Betrieb oder die Nationalität als Faktor von Einschluss/Ausschluss zu Arbeitsplätzen, die bereits angesprochen wurden.

Insgesamt handelt es sich um eine paradoxe Intervention: Ich benenne diese Gruppen mit dem Interesse, so Wechselwirkungen und Prozesse beschreiben zu können, während ich zugleich die Festschreibung von und Trennlinien zwischen Gruppen in der Einwanderungsgesellschaft hinterfrage.

Wenn es um Ungleichheit im Beruf geht, sind zwei Fragen wesentlich: die beruflichen vertikalen Hierarchien und die horizontale Segregation von Berufen nach Geschlecht und Migration. Zum einen wird Ungleichheit entlang der Hierarchien zwischen den einzelnen Berufsgruppen, also etwa zwischen Management und Büroarbeit oder zwischen ÄrztInnen und PflegerInnen, vertikal organisiert. Wie diese Beispiele beleuchten, stehen diese hierarchischen Gruppen in Wechselbeziehungen und nicht isoliert für sich. Denn sie formierten sich im Zusammenhang der ungleichen Arbeitsteilung entlang von Geschlecht und Migration. 
Zum Zweiten werden die meisten Berufe gegenwärtig nach Geschlecht/Migration horizontal - sozusagen nebeneinanderstehend - segregiert. Als ,weibliche“ Berufe gelten z. B. ErzieherInnen, FriseurInnen und andere soziale und persönliche Dienstleistungen. Demgegenüber werden industrielle Facharbeiter oder Automechaniker als ,männliche“ Berufe angesehen. Die Mehrheit der erwerbstätigen langheimischen Deutschen wie EinwanderInnen arbeiten in geschlechtssegregierten Berufen (BIBB 2016, S. 123 ff.). Die weiblich stereotypisierten Berufe bieten meist geringere Löhne und kaum Aufstiegsmöglichkeiten. Deswegen spricht man von horizontaler geschlechtlicher Segregation zwischen den Berufen.

Im Folgenden zeige ich die ungleichen Positionierungen nach Geschlecht und Migration in den hierarchischen Berufsrängen auf, indem ich die Verteilung der einzelnen Gruppen über diese Ränge vergleiche: Welcher Anteil unter den langheimisch deutschen oder den deutsch-türkischen Männern etwa gehört zu den Führungskräften (BetriebsleiterInnen, Management) und welcher zu Handwerk oder gelernter Industriearbeit? ${ }^{10}$ Auch wenn die Lektüre dadurch recht trocken gerät, werde ich die einzelnen Verteilungen mit Prozentangaben belegen, um so meine Aussagen überprüfbar darzulegen.

\subsection{Zunehmende (männliche) Diversität im beruflichen Spitzenfeld}

Ich werde nun zunächst auf die Führungskräfte und die akademischen Berufe eingehen, in denen sich die Teilhabe nach Geschlecht und Migration von 1996-2011 deutlich verändert hat. Im Mikrozensus wurden die Sparten der BetriebsleiterInnen aus Groß- und Kleinunternehmen und ManagerInnen ausgewertet. ${ }^{11}$ EinwanderInnen waren davon, soweit Angaben vorliegen, in den 1990er-Jahren weitgehend ausgeschlossen. Heute ist der Führungskräfte-Anteil unter südeuropäischen Männern mit deutschem Pass und Italienern gleich hoch wie unter langheimisch deutschen Männern: 2011 machten sie unter langheimisch deutschen Männern 8,6\%, unter deutschitalienischen $8,8 \%$, unter deutsch-exjugoslawischen $8,3 \%$, unter deutsch-türkischen $8,9 \%$ und unter italienischen $10,4 \%$ aus.

Dieses „Gleichziehen“ ist ein wichtiges und überraschendes Resultat. Es widerspricht dem heute noch tiefverwurzelten Stereotyp von Migranten als „Gastarbeitern“. Wie eine Feinanalyse ${ }^{12}$ der Untergruppen unter den Führungskräften zeigte, geht diese Veränderung sowohl auf Selbstständigkeit in der „,ethnischen Ökonomie“

\footnotetext{
10 Dazu wurde die Verteilung der einzelnen Gruppen über die Hauptberufsgruppen nach der ISCO-Berufsklassifikation 88 (International Standard Classification of Occupations), die dem Mikrozensus zugrunde gelegt wird, in Kreuztabellen ausgewertet. Bei den Feinanalysen wurde die Verteilung über deren Untergruppen ermittelt; so etwa in der Hauptgruppe ISCO 1 Führungskräfte die Untergruppen 111 Direktoren (...) 122 Produktions- und Operationsleiter und 130, 131 Leiter kleiner Unternehmen. Die ISCOKlassifikation ordnet die Berufsgruppen nach den inhaltlichen Tätigkeitsfeldern in Verbindung mit vier Qualifikationsstufen, die von der Primärausbildung bis zum Hochschulabschluss führen.

11 In der ISCO 88 Klassifikation (vgl. Fußnote 10) sind die Führungskräfte als Angehörige gesetzgebender Körperschaften, leitende Verwaltungsbedienstete und Führungskräfte in der Privatwirtschaft ausgewiesen. Ein Teil der soloselbstständigen Unternehmer ist unter prekären Bedingungen tätig, was jedoch weitere Untersuchungen erfordert.
}

12 Zur Feinanalyse vgl. Fußnote 10. 
(etwa von Pizzarestaurants) wie auch auf das Vorrücken der deutsch-südeuropäischen Männer ins Management zurück (Lenz 2020). Als Ursachen lassen sich die höhere Bedeutung des persönlichen Humankapitals im Zuge der Ökonomisierung und Technisierung der Lohnarbeit einerseits und die sichere Planungsperspektive, die sich mit der deutschen Staatsangehörigkeit verbindet, und gestiegene Bildungsgrade (vgl. Lenz 2020) andererseits vermuten.

Unter südeuropäischen Männern mit ausländischem Pass liegen die Führungskräfteanteile meist mit 5-6\% niedriger, sind aber von 1996-2011 angewachsen. Noch niedriger sind sie mit $3 \%$ unter Polen und 2,7\% unter Deutsch-Russen, wo ein langzeitlicher Ausschluss vorliegt.

Unter den Frauen aller Gruppen ist der Anteil von Führungskräften noch geringer und liegt teils nur bei der Hälfte der Männer. In anderen Worten sind sie in allen Gruppen von Führungspositionen ausgeschlossen.

Festzuhalten ist also, dass langheimisch deutsche Männer und deutsche Männer mit südeuropäischem Migrationshintergrund eine gleiche Teilhabe an den Führungskräften erreicht haben. Männer mit ausländischem Pass und Frauen sind weiterhin marginalisiert oder ausgeschlossen.

Der Anteil an akademischen Berufen wie Ärzte, Ingenieure, Rechtsanwälte usw. ist demgegenüber unter Frauen und Männern der jeweiligen Gruppe relativ gleich. Diese tendenziell gleiche Beteiligung trifft nur für die AkdemikerInnen zu. Unter langheimisch deutschen Männern liegt er bei $18 \%$, bei den russischen bei $15,2 \%$. Deutsch-Polen $(8,4 \%)$ und Deutsch-Türken $(7,5 \%)$ sind im Mittelfeld, während Türken (2,6\%) und Männer aus Ex-Jugoslawien (4,0\%) mit ausländischem Pass eher ausgeschlossen sind. Unter den Frauen umfassen die langheimisch deutschen und die mit Migrationshintergrund von 7,2\% bis 17,8\%. An der Spitze liegen die aus der GUS mit 17,8\%, dann folgen die langheimisch deutschen mit 14,8\%. Auch unter den Frauen bilden die Deutsch-Türkinnen $(8,1 \%)$ und die Deutsch-Polinnen $(7,2 \%)$ das Mittelfeld. Türkinnen (3,0\%) und Frauen aus Ex-Jugoslawien (3,1\%) mit ausländischem Pass sind fast ausgeschlossen. Der Migrationsstatus wirkt sich klar auf die Teilhabe an akademischen Berufen aus.

Wenn man die Anteile in Bezug auf Führungskräfte und akademische Berufe zusammenzählt, ergibt sich ein grober Indikator für die Inkorporation an der Spitze der beruflichen Hierarchie. Bei fast allen Gruppen sind die entsprechenden Anteile gestiegen. Mit einem Fünftel bis zu einem Viertel ist die Teilhabe hoch unter langheimisch deutschen Männern und deutsch-italienischen, deutsch-jugoslawischen und italienischen Frauen und Männern. Deutsch-türkische $(16,4 \%)$ und deutsch-polnische (13,3\%) Männer erreichen ein mittleres Niveau; bei den Frauen mit Migrationshintergrund liegt die Teilhabe unter der der Männer; sie schwankt zwischen $5 \%$ bis 22,4\%. Doch die EinwanderInnen mit ausländischem Pass aus Süd- und Osteuropa sind weiterhin stark unterrepräsentiert.

An der Spitze der beruflichen Hierarchien zeigt sich heute eine selektive Inkorporation anstelle des vorigen Ausschlusses: Sie ist durch einen Einschluss von deutschen Männern mit und ohne Migrationshintergrund (mit etwa gleichen Anteilen) unter den Führungskräften, einer wachsenden Teilhabe vieler Frauen bei akademischen Berufen wie auch durch einen weitgehenden Ausschluss von Frauen und Männern mit südosteuropäischem Pass gekennzeichnet. 


\subsection{Die Fachkräfte und Handwerker in der beruflichen Mitte}

Die Fachkräfte verfügen über mittlere Schulabschlüsse und eine weiterführende Fachausbildung wie etwa als Finanzfachkräfte oder als KrankenpflegerInnen. Teils handelt es sich um eher sozialkommunikative und existenzsichernd entlohnte Tätigkeiten mit interessanten Arbeitsinhalten.

Welche Positionierungen ergeben sich in diesen Berufsgruppen nach Geschlecht und Migration? Vor allem nach Geschlecht zeigen sich große Unterschiede in allen Gruppen: Die Fachberufe nehmen unter den Frauen aller Gruppen durchgehend höhere Anteile ein als unter den Männern. Hohe Anteile von 24-30\% zeigen sich bei Frauen mit deutschem Pass, nämlich langheimisch deutschen Frauen, DeutschPolinnen, Deutsch-Italienerinnen und Deutsch-Jugoslawinnen. ${ }^{13}$

Unter den Frauen aus allen anderen Gruppen, also Italienerinnen, Deutsch-Türkinnen und Türkinnen, Polinnen und Russinnen mit deutschen und GUS-Pässen beträgt die Positionierung in den Fachkräften zwischen etwa 15-20\%. Diese Anteile sind bei südeuropäischen Frauen mit ausländischem Pass seit 1996 deutlich angestiegen. Auch Männer aus einigen Gruppen mit deutschem Pass stehen in diesem Mittelfeld wie langheimisch Deutsche, Deutsch-Italiener und Deutsch-Jugoslawen. Eher marginalisiert sind hier Männer, so Polen, Russen und Türken, mit deutschen und ausländischen Pässen. So scheinen das (männliche) Geschlecht, Herkunftsregion und ausländischer Pass in Richtung Ausschluss aus den Fachberufen zusammenzuwirken.

Die Fachberufe erweisen sich als zweiter wesentlicher Aufstiegsweg für Frauen aller Gruppen unterhalb der akademischen Berufe. Frauen aus allen Gruppen sind von einem Sechstel bis zu einem Drittel in den Fachberufen und damit in der lohnarbeitenden Mitte angekommen: Das gilt für langheimische Deutsche wie für Einwanderinnen. Auch Frauen mit Migrationshintergrund werden nicht (mehr) pauschal ausgrenzt, sondern können Berufsbildung in Teilhabe verwandeln. Männer zeigen demgegenüber eine mittlere Inkorporation in einige Fachberufe wie technische, EDV- und Bankberufe, während sie in weiblich stereotypisierten Sparten ausgegrenzt sind.

Das Handwerk stellt demgegenüber für Männer aller Gruppen ein wichtiges mittleres Berufsfeld dar: Ein Fünftel bis ein Drittel der Männer ist in die Handwerkberufe inkorporiert, mit Ausnahme der Polen, unter denen der Anteil knapp unter der Hälfte liegt. Die Anteile sind meist etwas zurückgegangen. Die Frauen aller Gruppen sind demgegenüber faktisch ausgeschlossen. Es zeigt sich auch hier eine durchgehende geschlechtliche horizontale Segregation.

\subsection{Zwischen Verkaufen, Putzen und Pflegen: personale Dienstleistungen und Verkauf}

Die personalen Dienstleistungen bilden eine Mischkategorie, in die unterschiedliche Tätigkeiten eingeordnet werden. Einen Pol bildet der Verkauf und Handel in

${ }^{13}$ Da die Daten zu Deutschen mit Migrationshintergrund erst ab 2005 erhoben werden, können im Unterschied zu Personen mit ausländischem Pass längerfristige Veränderungen nicht erfasst werden. 
Läden oder auf dem Markt. Ein weiterer besteht aus der bezahlten Pflege und Versorgung ebenfalls in Betrieben oder zu Hause. Der dritte Schwerpunkt ist Arbeit in Hauswirtschaft und Gaststättengewerbe, also das bezahlte Kochen und Reinigen im öffentlichen oder häuslichen Bereich. Gemeinsam ist diesen Tätigkeiten, dass sie nach der Schule grundsätzlich eine berufliche Bildung erfordern, weiterhin aber auch, dass sie eher niedrig entlohnt und hochgradig feminisiert sind.

Um 2011 betrug der Frauenanteil unter den Beschäftigten im Bereich der personalen Dienstleistungen und dem Verkauf 75,8\%; diese Berufe sind also klar geschlechtlich segregiert. Unter den Frauen umfassten personale Dienstleistungen und Verkauf mit einem Fünftel (20,3\%) den zweitgrößten Bereich nach den Fachkräften (28,7\%, s. oben) und diese Anteile wuchsen meist von 1996 nur leicht an, während unter den Männern nur zwei bis etwa zehn Prozent dort arbeiten. Am höchsten sind die Anteile unter Frauen aus Südeuropa mit ausländischem Pass und Deutsch-Türkinnen mit jeweils etwa dreißig Prozent. Es folgen Deutsch-Italienerinnen, DeutschJugoslawinnen, Frauen aus Osteuropa und langheimisch deutsche Frauen mit dem niedrigsten Anteil von knapp zwanzig Prozent. Eine Feinanalyse in Bezug auf die Untergruppen zeigt: Fast alle Einwanderinnen sind in gleichem Umfang in Verkauf und Handel angekommen.

Die Anteile in der formellen Pflege- und Sorgearbeit sind mit um die fünf Prozent unter langheimisch deutschen und eingewanderten Frauen etwa gleich hoch. Das deutet nicht auf eine klare Unterschichtung nach Migration in der formellen Sorgearbeit hin. Jedoch erfasst der Mikrozensus die informelle und irreguläre Arbeit in der Carearbeit (vgl. u. a. Lutz 2018) nicht, die dabei einzubeziehen wäre.

Demgegenüber lagen die Anteile in Gaststätten oder im Haushalt unter Italienerinnen, Jugoslawinnen, Polinnen und Russinnen mit ausländischem Pass mit 9,5-12,5\% doppelt über dem Durchschnitt von 4,6\%. Einwanderinnen mit ausländischem Pass sind also deutlich überdurchschnittlich vertreten, was angesichts der Arbeitsbedingungen auf eine Unterschichtung hindeutet.

\subsection{Arbeiterberufe zwischen qualifizierter Industriearbeit und Einfacharbeit}

Der Arbeiteranteil unter den Berufsgruppen ist mit dem wirtschaftlichen Strukturwandel zu Dienstleistungen zurückgegangen und ein überwiegend männliches Feld. Im Mikrozensus finden sich zwei Angaben zu ArbeiterInnen: Einmal wird der Beschäftigungsstatus erfasst, wo Arbeiter mit Selbstständigen und Angestellten kontrastieren. Langheimisch deutsche Männer sind etwa zur Hälfte als Angestellte und zu dreißig Prozent als Arbeiter beschäftigt. Doch Männer mit Migrationshintergrund haben überwiegend den Beschäftigungsstatus Arbeiter. Bei osteuropäischen Männern mit deutschem Pass und südeuropäischen Männern sind dies weiterhin mehr als die Hälfte, allerdings sind diese Anteile seit 1996 deutlich zurückgegangen. Es zeigt sich eine fortbestehende Unterschichtung: Die langheimisch deutschen Männer steigen aus der Arbeiterschaft auf, während ein Großteil der Migranten weiter dort verortet wird.

Bei den Frauen wiederholt sich dieses Muster: Unter den langheimisch deutschen Frauen sind nur 12,4\% als Arbeiterinnen beschäftigt, aber unter den Türkinnen und den Deutsch-Russinnen etwa $40 \%$. 
Auch bei den Berufsgruppen werden ArbeiterInnen erfragt. Dabei ist nicht der Beschäftigungsstatus leitend, sondern es sind dies die Qualifikation und der Arbeitsinhalt. Der Mikrozensus unterteilt hier zwischen Maschinenbedienung mit Ausbildung, also Industriearbeit, und ungelernten Hilfsarbeiten.

Die verbleibende Industriearbeit ist ein überwiegend männliches Feld. Unter den Männern ist neben dem Geschlecht vor allem die Herkunftsregion wichtig: Die Anteile der Industriearbeit sind bei fast allen männlichen Einwanderern zurückgegangen und liegen um 2011 mit etwa einem Viertel am höchsten unter Russen und Russlanddeutschen sowie bei denen mit türkischem Pass, worauf die Deutsch-Türken folgen. Bei Italienern und Jugoslawen mit deutschem oder ausländischem Pass betragen sie etwa ein Sechstel und bei langheimisch Deutschen, Deutsch-Italienern und Polen sind sie unter einem Zehntel. Unter den Männern mit Migratonshintergrund aus Russland und der Türkei mit den höchsten Arbeiteranteilen in der Berufsstatistik liegen diese zwar nur bei etwa einem Viertel, sie sind mehr als doppelt so hoch als bei den langheimisch Deutschen, sodass sich ein klares Bild der Unterschichtung ergibt. Die Frauen sind faktisch ausgeschlossen.

Einfacharbeit ${ }^{14}$ umfasst die un- oder angelernten Tätigkeiten im Haushalt, in Wasch- und Reinigungsbetrieben und in der industriellen Fertigung. Allerdings bringen gerade EinwanderInnen oft höhere Qualifikationen mit, die dann ohne weitere Anerkennung vernutzt werden. Sie bilden den Kern des flexiblen, wenig gesicherten Segments auf dem Arbeitsmarkt.

In der Einfacharbeit zeigt sich eine dramatische Unterschichtung nach Geschlecht und Migrationsstatus: Unter Männern mit deutschem Pass schwanken die Anteile in diesem untersten Berufsrang zwischen 7,1\% unter den langheimisch Deutschen und bis zu 12,1\% bei den Deutsch-Türken. Einfacharbeit betrifft unter ihnen also etwa jeden Zehnten. Unter Männern mit ausländischem Pass sind die Anteile deutlich höher und betragen zwischen 14,8 bis 18,3\%, wobei sie seit 1996 eher gleich blieben. Jeder Sechste oder Fünfte von ihnen kommt weiterhin dort an, wo der klassische Platz der Arbeitsmigranten war.

Demgegenüber liegen nur bei zwei Gruppen von Frauen, nämlich unter langheimisch deutschen und deutschitalienischen, die Anteile der Einfacharbeit zwischen etwa acht bis zehn Prozent. Bei einer mittleren Gruppe vor allem mit deutschem Pass, Deutsch-Jugoslawinnen, Deutsch-Polinnen und Deutsch-Türkinnen bewegen sich diese Anteile immerhin zwischen einem Sechstel bis zu einem Viertel. Eine massive Unterschichtung zeigt sich bei Frauen mit ausländischem Pass aus Russland, Polen und der Türkei sowie bei Deutsch-Russinnen: Knapp ein Drittel verbleibt jeweils in der Einfacharbeit. ${ }^{15}$ In anderen Worten hat etwa ein Drittel der Einwanderinnen mit ausländischem Pass aus Russland, Polen und der Türkei nur Chancen auf wenig anerkannte, eher ungesicherte und gering bezahlte Jobs ohne Aufstiegsmög-

\footnotetext{
14 Die englische Fassung der ISCO-Berufsklassifikation 88 lautet „elementary occupations“. Die deutsche Version spricht von „Hilfsarbeitskräften“, doch handelt es sich nicht um „helfende“ zuarbeitende, sondern um als einfach definierte Tätigkeiten; vgl. zu Einfacharbeit u. a. Hirsch-Kreinsen und Minssen (2013, S. 176-177).

15 Aufgeschlüsselt betragen um 2011 die Anteile der Einfacharbeit bei Frauen mit ausländischem Pass aus den GUS-Staaten 30,5\%, aus Polen 29,9\% und aus der Türkei 32,5\% sowie unter Deutschrussinnen $29,9 \%$.
} 
lichkeiten. Fast jede Dritte von ihnen wird auf den unteren Rand der bezahlten Arbeit verwiesen, den langheimisch deutsche Frauen und Männer sowie Männer mit deutschem Pass weitgehend hinter sich gelassen haben. Auch bei diesen Frauen bleiben diese Anteile von 1996 bis 2011 eher konstant, was auf eine langfristig verankerte Unterschichtung verweist. Geschlecht und Migrationsstatus wirken ganz unten in der Berufshierarchie als gewaltiger Verstärker von Ausgrenzung und Marginalisierung.

Allerdings wird diese Unterschichtung nur durch die Berücksichtigung von Geschlecht, Herkunftsland und Migrationsstatus überhaupt wahrnehmbar. Denn wenn man allein das Geschlechterverhältnis in der Einfacharbeit betrachtet, erscheint es mit einem Frauenanteil von 52,4\% und einem Männeranteil von 47,6\% zunächst relativ ausgewogen. Erst in der Wechselwirkung von Geschlecht und Migrationsstatus zeigt sich diese Ausgrenzung von fast einem Drittel der Einwanderinnen mit ausländischem Pass aus Ost- und Südeuropa.

\section{Zum Schluss}

In diesem Artikel habe ich vertreten, dass die Transformation des Kapitalismus sich auf die intersektionalen Ungleichheiten auswirken kann. Darauf habe ich die parallel verlaufenden Veränderungen in den Berufshierarchien in Deutschland nach Geschlecht und Migration von 1996-2011 vorgestellt. Dieser Zeitrahmen nimmt zwar die neue Fluchtmigration seit 2015 nicht auf, dennoch zeigen sich klare Veränderungen in den Zugängen zu den Berufsrängen nach Geschlecht und Migration. Da große Einwanderergruppen berücksichtigt sind, sind diese Ergebnisse durchaus relevant für die Debatte um Intersektionalität. Ich nehme an, dass diese Veränderungen nicht mit starren dualistischen Ansätzen zu erfassen sind und plädiere deshalb für den Ansatz prozessualer Intersektionalität, also zu untersuchen, wie intersektionale Ungleichheitsverhältnisse sich verändern. Allerdings konnten hier nur einige Veränderungen deskriptiv festgehalten werden.

In diesen fünfzehn Jahren zeigte sich ein Wandel vom weitgehenden Ausschluss von EinwanderInnen aus Spitzenpositionen und mittleren Berufsrängen zu einer selektiven Inkorporation in die Spitze und Mitte. Zugleich wurden große, allerdings zurückgehende Gruppen weiterhin in die Arbeiterschaft segmentiert. Bei Migrantinnen mit ausländischem Pass trat eine dramatische Unterschichtung in die Einfacharbeit zutage. Allerdings konnte ich hier nur den Wandel selbst zusammenfassen, während dessen Ursachen weitere Forschungsfragen darstellen.

Diese Öffnung und Rekonfiguration in Bezug auf Migration vollzog sich im Rahmen einer zäh fortbestehenden durchgehenden Segmentation nach Geschlecht. Die Führungskräfte wurden nun ein diverses männliches Feld - die Krawatten werden bunter. Qualifizierte und einfache Dienstleistungen wurden weibliche diverse Felder. Während deutsche Männer tendenziell aus der Arbeiterschaft aufsteigen, ist der Anteil unter Migranten insbesondere mit ausländischem Pass zwar klar zurückgegangen, aber weiter bei etwa der Hälfte. Nur bei den akademischen Berufen zeigte sich ein Trend zu genereller gleicher Teilhabe, wobei in einzelnen Berufszweigen - etwa unter Ingenieuren oder LehrerInnen - wiederum stark geschlechtliche Segmentierungen auftreten. 
Nochmals hinweisen möchte ich auf die gewaltige Unterschichtung in die Einfacharbeit nach Geschlecht, Herkunftsregion und Migrationsstatus. Während in Westdeutschland das ,katholische Arbeitermädchen vom Land“ zur Zentralfigur intersektionaler Ungleichheit wurde, ist diese nach Ahmet Toprak heute ,muslimisch, männlich, desintegriert“ (Toprak 2019). Blicken wir jedoch auf die Berufschancen, so lautet der Befund eindeutig: ,migrantisch, osteuropäischer/südeuropäischer Pass, weiblich“. Trotz gestiegener Bildungschancen von Mädchen zeigt sich bei einem Drittel dieser Gruppen eine Unterschichtung in die Einfacharbeit. Da Frauen aus Russland, Polen und der Türkei gleich davon betroffen sind, ist das nicht auf den Islam zurückzuführen, was sonst oft bei migrantischen Problemlagen vermutet wird. Vielmehr sind das weibliche Geschlecht und der ausländische Migrationsstatus kennzeichnend.

Dass große Gruppen von Frauen so von weiterführenden Berufs- und Lebenschancen ausgeschlossen werden, findet kaum Aufmerksamkeit - im Gegensatz zu der kulturellen Debatte um Diskriminierung von Frauen aufgrund des Kopftuchtragens. Ohne Letztere relativieren zu wollen, zeigt sich hier eine kulturalistische Engführung in der intersektionalen Debatte, in der sozioökonomische strukturelle Ungleichheiten wenig wahrgenommen werden.

Ein weiteres wichtiges Ergebnis lautete, dass in Bezug auf die unterschiedlichen Berufschancen die Variable Migrationsstatus meist ähnlich bedeutend war wie die der Herkunftsregion (vgl. auch Söhn 2011). Deutsche mit Migrationshintergrund waren zur beruflichen Spitze vorgedrungen und auch in der lohnarbeitenden Mitte vertreten, während die meisten Gruppen mit ausländischem Pass von den Führungskräften ausgeschlossen und auch in der Mitte in geringerem Umfang positioniert waren. Der Ansatz der Inklusion/Exklusion diskutiert die einschließende Bedeutung von Citizenship, die institutionell soziale und ökonomische Chancen für die vermittelt, die Staatsbürger sind oder einen festen Aufenthaltstitel haben, während sie zugleich als nicht zugehörig identifizierte Personen ausschließt (Ataç und Rosenberger 2013). Die institutionelle Sicherung von Lebens- und Berufsplanung erwies sich auch in der Fallstudie als ein wichtiger, aber nicht alleiniger Aspekt für Chancen der Teilhabe.

Weiterhin ergibt sich daraus eine grundlegende methodologische Schlussfolgerung: Wenn die gegenwärtigen Veränderungen und Differenzierungen im Feld der Migration ermittelt werden sollen, ermöglichen die herkömmlichen Dualismen nach „Deutsche“/,Ausländer“ oder nur die Berücksichtigung der Herkunftsregion (PolInnen, TürkInnen usw.) keine angemessenen Zugänge. Es besteht die Gefahr, dass beträchtliche Unterschiede zwischen den Gruppen auf Durchschnitte nivelliert werden, die für alle betrachteten Gruppen wenig charakteristisch sind. Vielmehr sind mindestens die Migrationsgeschichte einschließlich des Migrationsstatus und der mitgebrachten und in Deutschland erworbenen Qualifikationen zu berücksichtigen.

Die Öffnungen nach Migration und Geschlecht in der beruflichen Spitze und Mitte, so lässt sich vermuten, erfolgen entsprechend den Impulsen und dem Druck des Marktes und der Ökonomisierung der Betriebe. Jedoch handelt es sich um selektive Inkorporation, also eine Aufnahme (oder eine Senkung der vorigen Schwellen bezüglich) hoch und mittel qualifizierter Einwanderinnen, während Ausschlüsse und Segmentierungen nach unten fortbestehen oder reorganisiert werden. Oben und in 
der Mitte zeigen sich Öffnungen, während weiter nach Geschlecht und ausländischem Pass in die schrumpfende Industriearbeit und Einfacharbeit segmentiert wird.

Diese Öffnungen sind auch das Ergebnis von jahrzehntelangem persönlichen Engagement gegen den vorigen Ausschluss und sozialer, insbesondere feministischer und antirassistischer Bewegungen. Berufliche Aufstiege von EinwanderInnen stützen sich nach empirischen Untersuchungen auf hohen persönlichen Einsatz, Unterstützung durch familiale und Freundschaftsnetzwerke und einzelne FörderInnen im sozialen Umfeld, während oft gewaltige institutionelle Widerstände in Schule und Betrieb zu überwinden waren (vgl. u. a. El-Mafaalani 2012; Lang et al. 2016). Sie stellen eine ungeheure, aber einstweilen unsichtbare Leistung dar, die eine entsprechende soziale Anerkennung erfordert.

Zugleich nehmen die Chancen zu, dass sich nun EinwanderInnen als ,organische Intellektuelle“ in die Debatten der postmigrantischen Gesellschaft (vgl. Foroutan et al. 2018) einmischen. Allerdings dürften die Konflikte mit der Diversität der Stimmen zunehmen (El-Mafaalani 2018). Die antirassistische Bewegung, die 2020 in Deutschland aufflammte, hatte viele SprecherInnen mit Migrationserfahrungen in akademischen Berufen und sie konnte trotz der Coronakrise viele jüngere migrantische und langheimisch deutsche Menschen mobilisieren (vgl. u.a. Zeit online 07.06.2020). Diese Entwicklung bedeutet eine grundlegende Demokratisierung durch Teilhabe von bisher marginalisierten MigrantInnen. Auch deswegen lautet die soziologische Frage nicht, ob diese Konflikte ,legitim“ sind, sondern wie sie verlaufen und soziologisch zu verstehen sind (El-Mafaalani 2018).

In ihnen zeichnen sich gegenwärtig mehrere gesellschaftspolitische Erzählungen ab. Eine Erzählung aus rechtskonservativer bis rechtsextremer Sicht beschwört nach wie vor das Gastarbeiterbild wenig gebildeter integrationsunwilliger MigrantInnen, die eine Belastung für die deutsche Gesellschaft darstellten. Während in diesem Rahmen nicht auf den damit verbundenen Rassismus eingegangen werden kann, zeigt diese Untersuchung, dass sie faktisch unbegründet ist und an der gesellschaftlichen Entwicklung vorbeigeht.

Eine weitere Erzählung behauptet den Vorrang der Klasse vor der von manchen sogenannten „Identitätspolitik“, in der Geschlecht und Migration privilegiert würden. Diese Untersuchung weist darauf hin, dass ein solcher Gegensatz wenig Sinn macht, da Klasse, Geschlecht und Migration etwa am Beispiel der Berufshierarchien erst in ihrer Wechselwirkung zu verstehen sind.

Schließlich argumentieren viele antirassistische SprecherInnen im Sinne positionaler Identität, dass MigrantInnen generell unterprivilegiert gegenüber langheimisch Deutschen seien. Das ergebe sich aus der Kategorie Migration oder Rasse, die als homogene, miteinander wechselwirkende Kategorien aufgefasst werden. Die Vorstellung homogener kategorialer Ausgrenzung und Unterordnung stimmt zumindest heute mit der komplexeren sozialen Wirklichkeit nicht überein. Denn all diese Kategorien sind intern nach Klasse oder Sexualität differenziert und die Differenzierungen zwischen EinwanderInnen oder auch Geschlechtern haben zugenommen. Eine generelle Unterprivilegierung ist zum Beispiel angesichts eines gleichen Anteils von Führungskräften unter deutsch-türkischen und langheimisch deutschen Männern empirisch nicht aufrechtzuerhalten. 
Konflikte über Teilhabe und Gleichheit in der Arbeit werden in postmigrantischen Gesellschaften zunehmen (El-Mafaalani 2018) und auch angesichts der Transformation des Kapitalismus werden neue Perspektiven und Lösungen erforderlich. Die Ungleichheitskategorien von Geschlecht und Migration haben sich differenziert und es zeigen sich komplexe intersektionale Konfigurationen. Eben hier liegen neue grundlegende Herausforderungen für eine intersektionale Ungleichheitsforschung.

Funding Open Access funding enabled and organized by Projekt DEAL.

Open Access Dieser Artikel wird unter der Creative Commons Namensnennung 4.0 International Lizenz veröffentlicht, welche die Nutzung, Vervielfältigung, Bearbeitung, Verbreitung und Wiedergabe in jeglichem Medium und Format erlaubt, sofern Sie den/die ursprünglichen Autor(en) und die Quelle ordnungsgemäß nennen, einen Link zur Creative Commons Lizenz beifügen und angeben, ob Änderungen vorgenommen wurden.

Die in diesem Artikel enthaltenen Bilder und sonstiges Drittmaterial unterliegen ebenfalls der genannten Creative Commons Lizenz, sofern sich aus der Abbildungslegende nichts anderes ergibt. Sofern das betreffende Material nicht unter der genannten Creative Commons Lizenz steht und die betreffende Handlung nicht nach gesetzlichen Vorschriften erlaubt ist, ist für die oben aufgeführten Weiterverwendungen des Materials die Einwilligung des jeweiligen Rechteinhabers einzuholen.

Weitere Details zur Lizenz entnehmen Sie bitte der Lizenzinformation auf http://creativecommons.org/ licenses/by/4.0/deed.de.

\section{Literatur}

Ataç, Ilker, und Sieglinde K. Rosenberger. 2013. Inklusion/Exklusion - ein relationales Konzept der Migrationsforschung. In Politik der Inklusion und Exklusion, Hrsg. Ilker Ataç, Sieglinde Rosenberger, 35-52. Göttingen: Vandenhoek \& Rupprecht.

Atzmüller, Roland, et al. 2015. Die zeitgemäße Arbeitskraft. Qualifiziert, aktiviert, polarisiert. Weinheim: Beltz Juventa.

Aulenbacher, Brigitte, et al. 2014. Sorge: Arbeit, Verhältnisse. Regime. Soziale Welt, Bd. 20 Sonderband. Baden-Baden: Nomos Verlag.

Aulenbacher, Brigitte, et al. 2015. Feministische Kapitalismuskritik. Münster: Westfälisches Dampfboot.

Bandorski, Sonja, et al. 2009. Der Mikrozensus im Schnittpunkt von Geschlecht und Migration: Möglichkeiten und Grenzen einer sekundär-analytischen Auswertung des Mikrozensus 2005; Berichtszeitraum 01.10.2006-15.12.2007. Baden-Baden: Nomos.

Becker, Karina, et al. (Hrsg.). 2020. Gespannte Arbeits- und Geschlechterverhältnisse im Marktkapitalismus. Wiesbaden: VS.

Becker-Schmidt, Regina, und Gudrun Axeli Knapp. 2000. Feministische Theorien zur Einführung. Hamburg: Junius.

BIBB (Bundesinstitut für Berufsbildung). 2016. Datenreport zum Berufsbildungsbericht 2016. Bonn: BIBB. Informationen und Analysen zur Entwicklung der beruflichen Bildung.

Crenshaw, Kimberlé. 1989. Demarginalizing the intersection of race and sex: a black feminist critique of antidiscrimination doctrine, feminist theory and antiracist politics. University of Chicago Legal Forum 139-167, https://chicagounbound.uchicago.edu/uclf/vol1989/iss1/8. Zugegriffen: 9. Nov. 2020.

Dietze, Gabriele. 2017. Sexualpolitik. Verflechtungen von Race und Gender. Frankfurt a.M.: Campus.

Dörre, Klaus, et al. (Hrsg.). 2012. Kapitalismustheorie und Arbeit. Neue Ansätze soziologischer Kritik. Frankfurt a.M.: Campus.

Edgell, Stephen, et al. (Hrsg.). 2016. The SAGE handbook of the sociology of work and employment. Los Angeles: SAGE.

El-Mafaalani, Aladin. 2012. BildungsaufsteigerInnen aus benachteiligten Milieus. Habitustransformation und soziale Mobilität bei Einheimischen und Türkeistämmigen. Wiesbaden: VS.

El-Mafaalani, Aladin. 2018. Das Integrationsparadox. Warum gelungene Integration zu mehr Konflikten führt. Köln: Kiepenheuer \& Witsch. 
Foroutan, Naika, Riem Spielhaus, und Juliane Karakayali (Hrsg.). 2018. Postmigrantische Perspektiven. Ordnungssysteme, Repräsentationen, Kritik. Frankfurt a.M.: Campus.

Ghaderi, Cinur. 2014. Politische Identität - Ethnizität - Geschlecht. Selbstverortungen politisch aktiver MigrantInnen. Studien zur Migrations- und Integrationspolitik. Wiesbaden: VS.

Gottfried, Heidi. 2013. Gender, Work, and Economy. Unpacking the Global Economy. Cambridge: Polity.

Gunda-Werner-Institut. 2019. „Reach Everyone on the Planet ...“: Kimberlé Crenshaw und die Intersektionalität. https://www.boell.de/de/2019/04/16/reach-everyone-planet. Zugegriffen: 8. Mai 2019.

Hark, Sabine, und Paula-Irene Villa. 2017. Unterscheiden und herrschen. Ein Essay zu den ambivalenten Verflechtungen von Rassismus, Sexismus und Feminismus in der Gegenwart. Bielefeld: transcript.

Hill Collins, Patricia, und Sirma Bilge. 2016. Intersectionality. New York: John Wiley \& Sons.

Hirsch-Kreinsen, Hartmut, und Heiner Minssen (Hrsg.). 2013. Lexikon der Arbeits- und Industriesoziologie. Berlin: Edition Sigma.

ILO. 2014. Profits and poverty. The economics of forced labour. Genf: ILO.

Klinger, Cornelia, Birgit Sauer, und Gudrun-Axeli Knapp (Hrsg.). 2007. Achsen der Ungleichheit. Zum Verhältnis von Klasse, Geschlecht und Ethnizität. Frankfurt a.M.: Campus.

Kocka, Jürgen. 2014. Geschichte des Kapitalismus. München: Beck.

Kößler, Reinhart. 2013. Kapitalismus und Moderne. Peripherie 130-131:149-178. http://www.linksnet.de/ de/artikel/29485. Zugegriffen: 26. Okt. 2020.

Lang, Christine, Andreas Pott, und Jens Schneider. 2016. Unwahrscheinlich erfolgreich. Sozialer Aufstieg in der Einwanderungsgesellschaft. IMIS Beiträge, Bd. 49 Universität Osnabrück.

Lenz, Ilse. 1995. Geschlecht, Herrschaft und internationale Ungleichheit. In Das Geschlechterverhältnis als Gegenstand der Sozialwissenschaften, Hrsg. Regina Becker-Schmidt, Gudrun Axeli Knapp, 19-47. Frankfurt a.M.: Campus.

Lenz, Ilse. 2010. Gender, inequality, and globalization. In Globalization and transformations of social inequality, Hrsg. Ulrike Schuerkens, 175-203. London: Routledge.

Lenz, Ilse. 2017. Genderflexer? Zum gegenwärtigen Wandel der Geschlechterordnung. In Geschlecht im flexibilisierten Kapitalismus. Neue UnGleichheiten, Hrsg. Ilse Lenz, et al., 181-222. Wiesbaden: VS.

Lenz, Ilse. 2018. Ungleichheiten nach Geschlecht und Migration in der postmigrantischen deutschen Gesellschaft. In Postmigrantische Perspektiven. Ordnungssysteme, Repräsentationen, Kritik, Hrsg. Naika Foroutan, et al., 129-144. Frankfurt a.M.: Campus.

Lenz, Ilse. 2019a. Intersektionale Konflikte in sozialen Bewegungen. Forschungsjournal Soziale Bewegungen 32(3):408-423. https://doi.org/10.1515/fjsb-2019-0046.

Lenz, Ilse. 2019b. Was kommt nach dem Patriarchat? Das Argument 330:826-841.

Lenz, Ilse. 2020. Einwanderung, Geschlecht, Zukunft? Wie Deutschland sich verändert. Opladen: Barbara Budrich.

Lenz, Ilse, et al. (Hrsg.) 2017. Geschlecht im flexibilisierten Kapitalismus. Neue UnGleichheiten. Wiesbaden: VS.

Lutz, Helma. 2018. Die Hinterbühne der Care-Arbeit. Transnationale Perspektiven auf Care-Migration im geteilten Europa. Weinheim: Beltz Juventa.

Lutz, Helma, und Anna Amelina. 2017. Gender, Migration, Transnationalisierung. Eine intersektionelle Einführung. Bielefeld: transcript.

Lutz, Helma, et al. (Hrsg.). 2013. Fokus Intersektionalität. Bewegungen und Verortungen eines vielschichtigen Konzeptes. Wiesbaden: VS.

Meyer, Katrin. 2017. Theorien der Intersektionalität zur Einführung. Hamburg: Junius.

Pries, Ludger. 2010. Erwerbsregulierung in einer globalisierten Welt. Wiesbaden: VS.

Reckwitz, Andreas. 2017. Die Gesellschaft der Singularitäten. Berlin: Suhrkamp.

Scheele, Alexandra, und Stefanie Wöhl (Hrsg.). 2018. Feminismus und Marxismus. Weinheim: Beltz.

Schwenken, Helena. 2018. Globale Migration. Zur Einführung. Hamburg: Junius.

Söhn, Janina. 2011. Rechtsstatus und Bildungschancen. Die staatliche Ungleichbehandlung von Migrantengruppen und ihre Konsequenzen. Wiesbaden: VS.

Toprak, Ahmet. 2019. Muslimisch, männlich, desintegriert: Was bei der Erziehung muslimischer Jungen schiefläuft. Düsseldorf: Econ.

Wallerstein, Immanuel, et al. 2013. Does capitalism have a future? Oxford: Oxford University Press.

Winker, Gabriele, und Nina Degele. 2009. Intersektionalität. Zur Analyse sozialer Ungleichheiten. Bielefeld: transcript. 
Ilse Lenz ist Prof. em. für Soziologie (Geschlecht, soziale Ungleichheit) an der Ruhr-Universität Bochum. Veröffentlichungen u. a.: Die Neue Frauenbewegung in Deutschland (2010). Wiesbaden; Evertz, Sabine, Ilse Lenz und Saida Ressel. 2017. Geschlecht im flexibilisierten Kapitalismus. Neue UnGleichheiten. Wiesbaden; Mae, Michiko und Ilse Lenz. 2020. Gleichheit, Differenz, Partizipation. Die Frauenbewegung in Japan. Wiesbaden. 